\title{
Xanthogranulomatous cholecystitis: A clinicopathological study from a tertiary care health institution
}

\author{
Kansakar PBS ${ }^{1}$, Rodrigues $\mathrm{G}^{2}$, Khan $\mathrm{SA}^{3}$ \\ ${ }^{1,2}$ Department of General Surgery, Kasturba Medical College, ${ }^{3}$ Faculty, Department of Pharmacy Practice, Manipal \\ College of Pharmaceutical Sciences, Manipal University, Manipal, India
}

\begin{abstract}
Background: Xanthogranulomatous cholecystitis is an unusual and destructive form of chronic cholecystitis and is indistinguishable from other forms of cholecystitis which makes preoperative diagnosis and surgery difficult.

Objectives: To review the demographic and clinical aspects of xanthogranulomatous cholecystitis; to study the possibility of preoperative diagnosis and to identify the causes for difficult surgery.

Materials and methods: All cases histopathologically diagnosed as xanthogranulomatous cholecystitis over a period of six years from October 1999 to September 2005 at Kasturba Medical College Hospital, Manipal, India were included in the study. Data of the patients was collected retro and prospectively.

Results: A total of 615 patients underwent cholecystectomy out of which $33(5.2 \%)$ were diagnosed to have xanthogranulomatous cholecystitis. Ultrasound abdomen showed gallbladder wall thickening in 19 (57.5\%) cases and gallstones in $32(96.9 \%)$ cases. Thirty $(90.9 \%)$ underwent open cholecystectomy. Gallbladder could be removed totally in $25(75.6 \%)$ cases whereas five $(15.2 \%)$ had to undergo partial cholecystectomy and in one patient, only cholecystostomy could be performed due to dense adhesions. Laparoscopic cholecystectomy was attempted in 11 patients but successful only in two patients with a conversion rate of $81.8 \%$. Postoperative wound infection was seen in five (15.1\%) patients and one $(3 \%)$ had minor biliary leak which was treated conservatively. Histologically, xanthogranulomatous cholecystitis was associated with malignancy in one $(3.03 \%)$ patient. There was no mortality.

Conclusion: Clinical presentation of xanthogranulomatous cholecystitis was indistinguishable from chronic cholecystitis. Ultrasonography may reveal only non specific findings of calculi and thickened gall bladder wall. Hence preoperative diagnosis is unlikely. Cholecystectomy was usually difficult owing to dense adhesions of gallbladder and Calot's triangle. Conversion rate of laparoscopic cholecystectomy is higher. Morbidity associated with surgery is significant.
\end{abstract}

Key words: Xanthogranulomatous cholecystitis, Cholecystectomy, Malignancy.

$\mathrm{X}$ anthogranulomatous cholecystitis (XGC) is an unusual and destructive form of chronic cholecystitis $^{1,2}$. It is an uncommon lesion which may form a tumor-like mass in inflamed gallbladders ${ }^{3}$. Preoperative diagnosis is difficult and is often mistaken for gall bladder cancer ${ }^{4}$. Despite much clinical experience, diagnosis and management of this disease remains suboptimal with an incidence of around $1-3 \%{ }^{4,5}$. Developing countries from South Asia give an incidence of around $5-13 \%^{1,6}$.

Present study was done to review the demographic and clinical aspects of XGC, to study the possibility of preoperative diagnosis and to identify the causes for difficult surgery.

\section{Materials and methods}

All cases histopathologically diagnosed as XGC over a period of six years from October 1999 to September 2005 at Kasturba Medical College Hospital, Manipal, India were included in the study. Data of the patients was collected retro and prospectively. The data was compiled in a proforma which included details of the patients, symptom analysis, clinical findings, investigations, operative details and postoperative complications. 


\section{Results}

A total of 615 patients underwent cholecystectomy over a period of six years. Histopathology of 33 patients showed XGC, out of which one was associated with malignancy. The duration from October 1999 to July 2004 was retrospective which included 28 cases of XGC and the duration from August 2004 to September 2005 was prospective which included five cases. The age range was between 30 and 80 years with a mean of 50 years in XGC whereas in rest of the cholecystectomy cases, the mean age of 46.37 years was observed. In $\mathrm{XGC}$, the age range most affected was between 31-40 years. Twenty one out of 33 patients $(63.6 \%)$ were males and 12 were females $(36.4 \%)$ in XGC where as out of 615 patients who had undergone cholecystectomy, 273 (44.4\%) were males and $342(55.6 \%)$ were females. Thirty two patients $(97 \%)$ had history of pain abdomen. In 21 patients, pain was located in the right hypochondrium, in six both in the epigastrium and right hypochondrium, in four patients in the epigastrium alone and one presented with loin pain. In nine patients, there was history of jaundice and vomiting and eight had history of fever. Liver function tests showed increased total as well as direct bilirubin in four $(16 \%)$ out of 25 patients. Elevated alkaline phosphatase was observed in $19(65.5 \%)$ cases. Ultrasonography (USG) finding was as mentioned in Figure 1.

Out of 33 patients, 30 (90.9\%) underwent open cholecystectomy. Gallbladder could be removed totally in 25 patients $(75.6 \%)$ whereas five patients $(15.2 \%)$ had to undergo partial cholecystectomy. In one patient, only cholecystostomy could be performed due to dense adhesions. Laparoscopic cholecystectomy was attempted in 11 patients but was successful only in two patients with a conversion rate of $81.8 \%$. Antegrade cholecystectomy was performed in 18 patients $(54.5 \%)$ and retrograde cholecystectomy was performed in 14 (42.4\%). Common bile duct exploration with T-tube insertion was performed in five patients (15.2\%). Per operative findings are mentioned in Table 1.

Post operative wound infection developed in five patients $(15.1 \%)$ and one patient $(3 \%)$ had minor biliary leak which was treated conservatively. There was no mortality. Histopathologically, 24 (72.7\%) gall bladder specimens were reported as chronic XGC and remaining nine $(27.2 \%)$ as acute on chronic XGC. Grossly, gall bladder wall thickening was seen in all 33 patients $(100 \%)$. Gall bladder wall perforation was present in five patients (15.2\%). In 17 patients $(51.55 \%)$, yellowish discoloration was seen in the mucosal surface indicating xanthomatous change. Nodules were seen in eight patients $(24.2 \%)$ and polyps in three $(9.1 \%)$.

Microscopically, ulceration was seen in 22 specimens (66.7\%) and micro abscesses in three specimens (9.1\%). Foam cells characteristic of XGC were seen in all 33 gallbladder specimens. Foreign body giant cells were seen in 20 patients $(60.6 \%)$ and peri portal fibrosis in five $(15.2 \%)$. In one patient $(3 \%)$, XGC was associated with malignancy.

Table 1: Per operative findings of XGC

\begin{tabular}{|l|l|c|}
\hline \multicolumn{2}{|l|}{ Findings } & Number \\
\hline \multirow{5}{*}{ Gallbladder } & Distended & 9 \\
\cline { 2 - 3 } & Shrunken & 16 \\
\cline { 2 - 3 } & Not Visualized & 1 \\
\cline { 2 - 3 } & Wall thickening & 30 \\
\cline { 2 - 3 } & Gall bladder growth & 3 \\
\cline { 2 - 3 } & Pus & 6 \\
\hline CBD dilatation & Gall bladder & 6 \\
\hline \multirow{4}{*}{ Calculi } & CBD & 26 \\
\cline { 2 - 3 } & Gall bladder + CBD & 2 \\
\cline { 2 - 3 } & None & 2 \\
\hline Dense Adhesions & & 20 \\
\hline Mirizzi's syndrome & & 3 \\
\hline
\end{tabular}




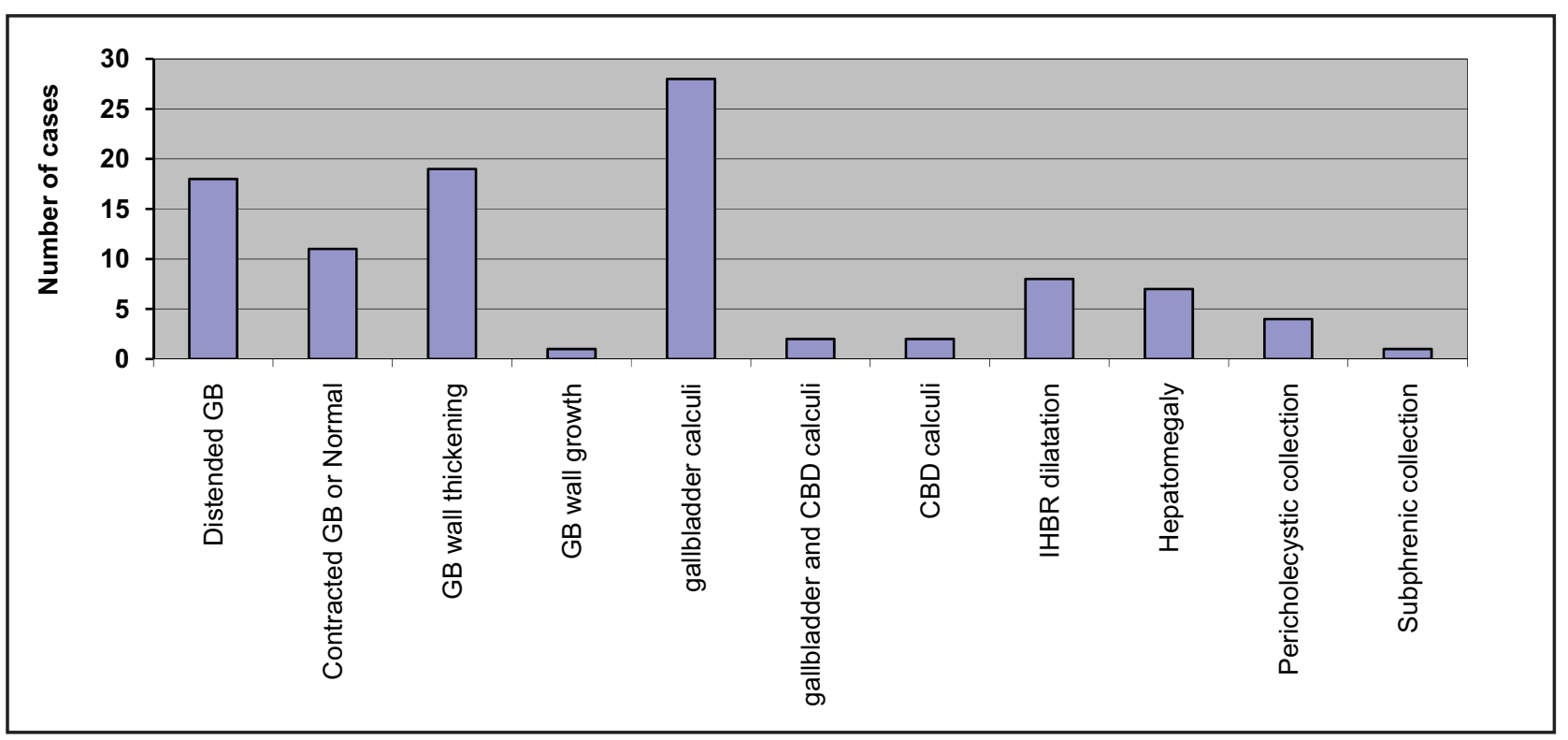

Fig 1: Ultrasonography findings of XGC

\section{Discussion}

Incidence of XGC was comparable with other series ${ }^{7}$ however it was considerably low as compared to series from developing countries like India ${ }^{8,9}$. This may be due to difference in climate and dietary habits wherein North Indian study showed higher incidence as compared to our study conducted in South India. Mean age of patients with XGC was 50 years that was comparable with other series ${ }^{7,9}$.

There was an association with gall stone disease in $96.9 \%$ of cases. It was comparable with the findings from Western ${ }^{7,10}$ and Eastern ${ }^{9}$ studies where a total of $84 \%$ to $100 \%$ cases were associated with gall stone disease. However it was high as compared to an Indian study where an incidence of $71.4 \%$ were reported ${ }^{8}$. USG showed thickened gallbladder wall in 57.6\% cases comparable with other series ${ }^{10}$. Three $(9.2 \%)$ cases were suspected to have gall bladder carcinoma but only one $(3.03 \%)$ turned out to be malignant which was comparable with a similar series in the literature ${ }^{7}$. Laparoscopic cholecystectomy was attempted in 11 patients in the present study and converted to open cholecystectomy in nine patients with a conversion rate of $81.8 \%$. This again was comparable to the same study, where $80 \%$ conversion rate has been reported ${ }^{7}$. Morbidity of surgery was $18.2 \%$ in the present study which was high as compared to other series and there was no mortality as compared to similar series ${ }^{7}$. Major reason for high morbidity was the high incidence of post operative wound infection in our institution ${ }^{11}$ as compared to other studies ${ }^{10}$.

\section{Conclusions}

$\mathrm{XGC}$ is an increasingly recognized but an infrequent form of chronic cholecystitis with an incidence of 5.2\% of all excised gall bladders. Clinical presentation of $\mathrm{XGC}$ is indistinguishable from chronic cholecystitis but incidence of jaundice and palpable mass in the right upper quadrant is higher. USG may reveal only non specific findings of calculi and thickened gall bladder wall. Hence pre operative diagnosis is unlikely. XGC can mimic carcinoma of gall bladder both radiologically and intra operatively but carcinoma of gall bladder also appears to be more frequently associated with XGC. In cases of suspicious malignancy, frozen section would be advisable as cholecystectomy would be an adequate treatment if XGC is diagnosed. Cholecystectomy is usually difficult owing to dense adhesions between gall bladder and surrounding structures with distorted anatomy. Conversion rate of laparoscopic cholecystectomy is higher. Morbidity associated with surgery is higher.

\section{References}

1. Rao RV, Kumar A, Sikora SS, Saxena R, Kapoor VK. Xanthogranulomatous cholecystitis: differentiation from associated gall bladder carcinoma. Trop Gastroenterol. 2005;26:31-3.

2. Kwon AH, Matsui Y, Uemura Y. Surgical procedures and histopathologic findings for patients with xanthogranulomatouscholecystitis. J Am Coll Surg. 2004;199:204-10.

3. Goodman ZD, Ishak KG. Xanthogranulomatous cholecystitis. Am J Surg Pathol. 1981;5:653-9. 
4. Cardenas-Lailson LE, Torres-GomezB, MedinaSanchez S, Mijares-Garcia JM, HernandezCalleros. Cir Cir. 2005;73:19-23.

5. Guzman-Valdivia G. Xanthogranulomatous cholecystitis in laparoscopic surgery. J Gastrointest Surg. 2005;9:494-7.

6. Budhiraja S, Wig JD, Gupta NM, Khanna SK. J Indian Med Assoc 2003;101:586-7.

7. Gilberto Guzman-Valdivia: Xanthogranulomatous cholecystitis. An experience of 15 years. World J Surg. 2004;8:254-7.

8. Solanki RL, Arora HL, Gaur SK, Anand VK, Gupta R. Xanthogranulomatous cholecystitis: Clinicopathological study of 21 cases. Indian J Pathol Microbiol. 1989;32:256-60.
9. SinghUR,AgarwalS, MisraK. Histopathological study of xanthogranulomatous cholecystitis. Indian J Med Res. 1989;90:285-8.

10. Houston JP, Collins MC, Cameron I, Reed MWR, Parsons MA, Roberts KM. Xanthogranulomatous cholecystitis. Br J Surg. 1994;81:1030-2.

11. Khan SA, Rao PGM, Rao A, Rodrigues G. Survey and evaluation of antibiotic prophylaxis usage in surgery wards of tertiary level institution before and after the implementation of clinical guidelines. Indian J Surg. 2006;68:150-6. 